\title{
Plataforma RELIEF
}

\author{
Renato Teixeira Vargas \\ NEP Consultoria
}

\begin{abstract}
RELIEF Platform - Structural Analysis Reference Center based on the Finite Element Method -, is a novel initiative in Brazil responsible for the technology of structural analysis transference to small and medium companies (SMC). The obstacles that do not allow introducing this technology are the lack of specialized engineers and the high cost of simulation tools. With this Platform, we intend to facilitate an efficient structural analysis technology transfer to the innovation process of SMCs. RELIEF is free of charge.
\end{abstract}

This Reference Center is a virtual platform which makes courses and tutorials based on CAE (Computer Aided Engineering) free software available. The proposed courses are synthesis between theoretical fundamentals and practical applications. The videoclasses were prepared by USP and Unicamp laboratory researchers, and companies. Three modules are available: Fundamentals, Workshops and Context. Fundamentals Module shows theoretical contents in Solid Mechanics and Finite Element Method disciplines. Workshops were introduced by experienced companies in the market. Context Module is composed by interviews with teachers, consultants and entrepreneurs in CAE area. RELIEF is operational.

\section{RESUMO}

Este artigo descreve a Plataforma RELIEF - Centro de Referência em Análise Estrutural Por Elementos Finitos uma Plataforma virtual gratuita criada para realizar a transferência da tecnologia de cálculo de estruturas, através de cursos teóricos e oficinas de aplicação prática, estabelecendo um ambiente para formação de engenheiros, especialmente provenientes de PMEs [1]. A tecnologia de análise estrutural contemplada no RELIEF está baseada no MEF, implementado em programas livres de engenharia assistida por computador (CAE). Esta iniciativa é uma inovação no Brasil, porque ainda não existem alternativas técnicas e financeiras viáveis para formação de pessoas e aquisição de ferramentas de cálculo para este segmento industrial no mercado. O RELIEF está dividido em 03 módulos. O Módulo FUNDAMENTOS fornece um acervo de conhecimentos nas disciplinas de mecânica dos sólidos teórica / experimental e MEF, com exercícios analíticos e de simulação numérica em programas livres. Os cursos deste Módulo foram criados em parceria com laboratórios da USP e da Unicamp. O Módulo OFICINAS disponibiliza videoaulas sobre modelagem e aplicação de ferramentas de cálculo na solução de problemas de Engenharia como: Automobilística, Óleo \& Gás, Polímeros, Estruturas Metálicas e Tubulações. O módulo CONTEXTO foi desenvolvido por meio de entrevistas com consultores e empresários experientes no mercado nacional. Após a realização do projeto Piloto no período entre novembro (2019) e março (2020), a Plataforma está em funcionamento desde março de 2020. O RELIEF foi financiado por meio de recursos do NEP Consultoria e do Projeto Pipe-Fapesp - Criação do Centro de Referência em Análise Estrutural - Processo 2017-20646-3 (01/02/19 a 31/10/1) [2].

\section{INTRODUÇÃO}

O Centro de Referência em Análise Estrutural por Elementos Finitos (RELIEF) é uma plataforma virtual que desenvolve, organiza e disponibiliza os fundamentos para a formação de um engenheiro na área de análise estrutural baseada no MEF: cursos teóricos, oficinas para solução de problemas práticos e a articulação deste conhecimento no contexto nacional.

O RELIEF pode ser classificado como uma inovação disruptiva, processo pelo qual um produto ou serviço inicialmente é ancorado a aplicações simples, mas pouco a pouco vai se movendo para fatias mais valorizadas do mercado [3]. As atividades da Plataforma estão orientadas para a criação dos cursos e material didático, e, gradativamente deverá priorizar a disponibilização dos cursos para a solução de problemas práticos nas empresas. A Plataforma RELIEF está inserida em um modelo de negócio classificado como Grátis [4], e garante a gratuidade das atividades essenciais da Plataforma. 
A construção da Plataforma foi financiada por meio de recursos de Projeto PIPE - Fapesp e da empresa NEP Núcleo de Consultoria em Engenharia e Pesquisa em tecnologia. Uma parte significativa dos trabalhos foram realizados por meio de Acordos de Cooperação Técnica com os professores e consultores responsáveis pela execução e manutenção do conteúdo.

O RELIEF passou por um período de Projeto Piloto e está disponível ao público desde março de 2020.

\section{A PROPOSTA DO RELIEF}

O RELIEF é uma plataforma pública e gratuita para viabilizar a transferência de tecnologia CAE e promover a inclusão das PMEs no mercado sob o paradigma da inovação. O RELIEF foi concebido para democratizar esta tecnologia entre as PMEs por meio de duas iniciativas: formação de técnicos e engenheiros, sem investimentos, e a utilização dos programas livres em substituição aos programas comerciais/restritos. A organização do conhecimento contemplou o equilíbrio entre as abordagens teórica e prática e foi construído em base cooperativa com universidades e empresas. Também foi abordado o contexto político e socioeconômico do país para estabelecer o diálogo entre a evolução desta tecnologia com a realidade do Brasil. O RELIEF está dividido em 03 módulos:

- Módulo FUNDAMENTOS - apresenta as disciplinas de mecânica dos sólidos teórica e experimental e do MEF. Este conteúdo é acompanhado de uma série de exercícios analíticos e de simulação numérica em programas livres. A comparação entre as soluções analítica/numérica/experimental é a base da metodologia de ensino da Plataforma. Estes cursos foram criados em parceria com laboratórios da USP (Grupo de Mecânica dos Sólidos e Impacto em Estruturas - GMSIE) e da Unicamp (Laboratório de Simulação Numérica), reconhecidos em nível internacional.

- Módulo OFICINAS - O módulo de OFICINAS disponibiliza videoaulas criadas por empresas e profissionais com mais de 20 anos de experiência na solução de problemas de Engenharia. Estão disponíveis oficinas nas áreas: Automobilística, Óleo \& Gás, Estruturas Metálicas, Tubulações e Polímeros. São apresentados tópicos sobre a programas de cálculo para a solução de problemas de Engenharia, mercado de trabalho e generalidades sobre consultorias: elaboração da parte comercial, definição de escopo, execução de um cronograma físico-financeiro, aspectos técnicos e elaboração de soluções.

- Módulo CONTEXTO - foi desenvolvido por meio de entrevistas com profissionais experientes no mercado nacional para explorar as trajetórias profissionais em diálogo contínuo com o contexto nacional.
A empresa Núcleo de Consultoria em Engenharia e Pesquisas em Tecnologia - NEP - é uma empresa componente do Diretório de Instituições de Pesquisa do $\mathrm{CNPq}$ e foi parceira no desenvolvimento Plataforma. O NEP (http://www.nepconsult.com.br/) atua há 15 anos na solução de consultorias e promoção de cursos sobre temas relativos à área de simulação de análises estruturais pelo MEF. O NEP financiou parte do desenvolvimento da Plataforma e é a responsável pelos custos de manutenção. Também desenvolveu as oficinas de Análise Estrutural e o MEF e Automobilística.

\section{A PLATAFORMA RELIEF}

A Plataforma disponibiliza cursos, oficinas, exercícios, entrevistas e programas CAE livres para consolidar uma base de informações que permita a capacitação de engenheiros para a solução de problemas de análise estrutural por meio do MEF. A premissa fundamental para a criação do RELIEF é a de que o engenheiro qualificado para exercer as atividades de análise estrutural deve conhecer os fundamentos teóricos das disciplinas, mas permanecer conectado às aplicações de engenharia. Todos os cursos e entrevistas foram gravados exclusivamente para o RELIEF. Este fato é importante e diferenciado porque é frequente a disponibilização de videoaulas na web, mas gravadas durante os cursos regulares. Esta prática não permite atingir um bom padrão de qualidade porque há limitações nas gravações durante um curso regular que impedem a aquisição da qualidade estabelecida para este projeto.

A Plataforma foi desenvolvida por uma equipe formada por 4 professores doutores, 5 engenheiros consultores com mais de 20 anos de experiência, 1 cinegrafista, 1 designer gráfico e 3 editores.

\section{MÓDULO FUNDAMENTOS}

O módulo FUNDAMENTOS apresenta as disciplinas de mecânica dos sólidos teórica e experimental e do MEF. Este conteúdo é acompanhado de uma série de exercícios analíticos e de simulação numérica em programas livres. Os cursos foram criados em parceria com laboratórios da USP (Grupo de Mecânica dos Sólidos e Impacto em Estruturas GMSIE) e da Unicamp (Laboratório de Simulação Numérica). A disciplina de Mecânica dos Sólidos foi ministrada desenvolvida pelo prof. Dr. Marcílio Alves [5], responsável pelo Grupo de Mecânica dos Sólidos e Impacto em Estruturas ((http://www.gmsie.usp.br) e gravadas nas instalações do laboratório.

As aulas sobre o MEF foram desenvolvidas pelo prof. Dr. Marco Lúcio Bittencourt [6], professor titular da Faculdade de Engenharia Mecânica da Unicamp. Ele é o responsável pelo Laboratório de Simulação Computacional, e foi componente do CONSICAE (FAPESP) - Consórcio 
Setorial para Engenharia Assistida por Computador entre 2004 e 2010 na linha de financiamento ConSITec Consórcios Setoriais para Inovação Tecnológica em colaboração com a Faculdade e empresas Mahle-Metal Leve, Embraco e Thyssenkrupp. As aulas foram gravadas nas "salas do curso básico" da Engenharia da Unicamp. As parcerias com USP e Unicamp foram viabilizadas por meio de Acordos e Propriedade Intelectual (API).

PROGRAMAS LIVRES - A Plataforma RELIEF também é inovadora porque propõe a utilização de programas de CAE livres no apoio aos cursos com exemplos e tutoriais, em uma perspectiva de uso destas ferramentas para a solução dos problemas reais nas empresas. Os programas livres apresentam algumas vantagens sobre os programas comerciais-restritos. Há vários programas livres de CAE, com diferentes abrangências nas soluções e qualidade da interface. Para a construção dos exercícios tutoriais do RELIEF foram utilizados 04 programas:

- Code Aster / SALOME - Meca: programa livre de análise estrutural 3D. Foi criado há 25 anos pela Companhia de Eletricidade da França (EDF) sob licença LGPL, e mantém uma equipe no desenvolvimento e manutenção. Este programa apresenta soluções em todas as áreas necessárias para análise estrutural linear e não linear: Estática Linear e Não Linear, Fadiga, Mecânica da fratura, Mecânica do Dano, Otimização, NVH, Multi-Corpos, e acoplamento térmico. O Code Aster realiza encontros regulares de usuários e disponibiliza atualizações anuais do programa. $\mathrm{O}$ programa é adotado por empresas como: AirBus, EDF, Valeo e universidades de importância mundial como o Imperial College, Escole Polytechnique. O Code Aster preferencialmente utiliza o programa Salome para o trabalho de pré e pós-processamento. O Code Aster e o Salome oferecem uma solução chamada Salome-MECA que habilita análises estáticas lineares, análise modal e de fratura por meio de interface semelhante aos programas restritos.

- Calculix: é um programa com módulos de solução para problemas lineares e não lineares estáticos e dinâmicos associados à temperatura. A interface do Calculix é simplificada, mas permite o pós-processamento dos resultados.

- Smath: é um programa matemático gratuito, com capacidade para resolução de equações diferenciais, e criação de funções gráficas em 2 ou 3 dimensões.

- Abaqus: programa restrito para validar as respostas dos programas livres.

MECÂNICA DOS SÓLIDOS TEÓRICA E EXPERIMENTAL - Descrição do Curso: Proporcionar uma visão ampla sobre a mecânica dos sólidos teórica e experimental para sedimentar conceitos de tensão e deformação e análises lineares estáticas e dinâmicas. Os conceitos de estática são ilustrados por meio de um ensaio de tração, realizado no laboratório do GMSIE. São introduzidos conceitos de dinâmica linear, consolidados em experimento de análise modal. É apresentada uma introdução à não-linearidade, com experimentos de impacto sobre tubos. Duração: 8 h. Professor: Prof. Dr. Marcílio Alves

Aula 1 - Conceito de Tensão e Deformação

Aula 2 - Leis Constitutivas

Aula 3 - Conservação de Volume

Aula 4 - Ensaio de Tração

Aula 5 - Ensaio de Tração - Experimental

Aula 6 - Dinâmica de Barras

Aula 7 - Análise Modal de Barras

Aula 8 - Equilíbrio de Vigas

Aula 9 - Dinâmica de Vigas

Aula 10 - Análise Modal de Vigas

Aula 11 - Análise Modal de Vigas - Experimental

Aula 12 - Princípio dos Trabalhos Virtuais - Vigas

Aula 13 - Método dos Elementos Finitos - Vigas

Aula 14 - Impacto em Tubos - Teoria

Aula 15 - Flambagem de Tubos - Experimental

Aula 16 - Flambagem Elástica

MÉTODO DOS ELEMENTOS FINITOS - Descrição do Curso: Apresenta os fundamentos teóricos do MEF. São enfatizados aspectos relativos à análise matricial, à formulação de elementos de barra e de viga, montagem de matriz de rigidez, transformação de coordenadas e diferentes formas de equilíbrio. Os tópicos são sedimentados por meio da proposição de exercícios com resolução anexada. Duração: 8 h. Professor: Prof. Dr. Marco Lúcio Bittencourt

Aula 1 - Elementos de Simulação I

Aula 2 - Elementos de Simulação II

Aula 3 - Conceitos do Método dos Elementos Finitos

Aula 4 - Formulação de Elemento de Barra I

Aula 5 - Formulação de Elemento de Barra II

Aula 6 - Cálculo de Erro

Aula 7 - Transformação de Coordenadas

Aula 8 - Exemplo: Treliça -Parte 1

Aula 9 - Exemplo: Treliça -Parte 2

Aula 10 - Formulação de Elementos de Viga

Aula 11 - Exemplo: Elemento de Viga - Parte 1

Aula 12 - Exemplo: Elemento de Viga - Parte 2

Aula 13 - Formulação de Elemento de Pórtico I

Aula 14 - Formulação de Elemento de Pórtico II

Aula 15 - Forma Forte de Equilíbrio I

Aula 16 - Forma Forte de Equilíbrio II

Aula 17 - Forma Fraca de Equilíbrio e o MEF I

Aula 18 - Forma Fraca de Equilíbrio e o MEF II

Aula 19 - Formulação de Elemento de Barra de 3 Nós

EXERCÍCIOS TUTORIAIS - Os cursos do Módulo FUNDAMENTOS foram ilustrados com exercícios analíticos e numéricos em programas livres. Os exercícios 
tutoriais foram desenvolvidos com o objetivo de sedimentar os conhecimentos teóricos transmitidos nas videoaulas dos cursos teóricos de Mecânica dos Sólidos e Método dos Elementos Finitos, introduzir a manipulação de programas CAE e disseminar a cultura de utilização de programas livres na área de análise estrutural. Nestes exercícios foram abordadas as áreas de análise estática linear e não-linear, dinâmica, flambagem. Alguns exercícios foram realizados por meio do cálculo analítico e comparados com soluções numéricas e experimentais. A equipe do RELIEF desenvolveu 2 programas para solução de problemas de viga por meio do método de diferenças finitas e que estão disponíveis na plataforma. Também foi realizada a comparação com um programa restrito (ABAQUS) para apresentar aos usuários a eficiência e a credibilidade dos programas livres - um fator essencial para assegurar às empresas e usuários a efetividade destes programas na solução de problemas práticos. Todo os exercícios são apresentados por meio de tutoriais passo-a-passo para permitir que um iniciante possa executar uma análise estrutural em todas as etapas da tecnologia CAE. Abaixo estão relacionados os exercícios:

Exercício 1 - Membros Carregados Axialmente

Treliça isostática

Ensaio de tração

Exercício 2 - Vigas

Vigas esbeltas e rombudas

Modelos de Euler e Timoshenko

Pórtico hiperestático

Exercício 3 - Eixos

Torção de eixos circulares

Torção de eixos retangulares

Exercício 4 - Flambagem

Flambagem elástica de colunas

Exercício 5 - Plasticidade

Ensaio de tração com plasticidade

Exercício 6 - Dinâmica I

Modos de Vibrar de Barras

Exercício 7 - Dinâmica II

Modos de Vibrar de Vigas

Exercício 8 - Dinâmica III

Análise modal de viga engastada/biapoiada

Exercício 9 - Dinâmica IV

Análise modal de pórtico hiperestático

\section{MÓDULO OFICINAS}

O módulo de OFICINAS disponibiliza videoaulas, especialmente criadas para o RELIEF, por empresas e profissionais com mais de 20 anos de experiência na solução de problemas de Engenharia. Estão disponíveis oficinas nas áreas: Automobilística, Óleo \& Gás, Estruturas Metálicas, Tubulações e Polímeros. São apresentados tópicos sobre a programas de cálculo para a solução de problemas de Engenharia, mercado de trabalho e generalidades sobre consultorias: elaboração da parte comercial, definição de escopo, execução de um cronograma físico-financeiro, aspectos técnicos e elaboração de soluções. Também são apresentadas características dos programas de elementos finitos livres e restritos e considerações sobre a disciplina de modelagem aplicada à ferramenta $\mathrm{CAE}$. O convênio entre as partes desenvolvedores das oficinas e proponente do projeto para a etapa de criação dos cursos foi formalizado por meio de Acordos de Cooperação Técnica, que acordam a formatação dos cursos, atribuições das partes, aspectos técnicos, direito de uso e divulgação.

ANÁLISE ESTRUTURAL E O MEF - São apresentados tópicos sobre a programas de cálculo para a solução de problemas de Engenharia, mercado de trabalho e generalidades sobre consultorias: elaboração da parte comercial, definição de escopo, execução de um cronograma físico-financeiro, aspectos técnicos e elaboração de soluções. Também são apresentados programas de elementos finitos livres e restritos. Duração: 4 h. Professor: Prof. Dr. Renato Vargas

Aula 1 - Considerações Sobre Análise Estrutural Métodos de Análise Estrutural

Um pouco de história

Bibliografia

Aula 2 - Considerações Sobre o MEF

Porque utilizar o MEF

Grupos de Pesquisa

Cursos de MEF e Tecnologia CAE

Tendências

Bibliografia

Aula 3 - Programas CAE

Qual é o melhor programa CAE

Arquitetura de um programa $\mathrm{CAE}$

Programas CAE restritos / Programas CAE livres

Programas CAD e de Geração de Malhas Restritos - Livres

Aula 4 - Análises Estruturais Utilizando Programas CAE

Descrição de casos reais:

Válvula industrial

Roda de plástico

Aula 5 - Generalidades sobre consultorias

Definição de escopo - identificar desafios

Elaboração da proposta comercial

Execução do cronograma físico-financeiro

Diferenças - Empresas Privadas e Públicas

Mercado de trabalho - tendências e perspectivas

POLÍMEROS - Apresentar as principais peculiaridades de análise estrutural dos plásticos. A sofisticação dos materiais poliméricos e o seu uso intensivo e crescente impõe o conhecimento da variação de seu comportamento mecânico segundo a influência da temperatura, umidade, fluência, fadiga, exposição à UV e envelhecimento. Por outro lado, os diferentes processos de fabricação dos plásticos como injeção, extrusão, sopro e a 
adição de reforços e aditivos introduzem variações significativas nas propriedades mecânicas. A modelagem numérica deve reproduzir esta complexidade e o analista deve estar consciente das aproximações necessárias para realizar uma avaliação realista da performance do produto em acordo com as potencialidades dos programas CAE. Duração: 5 h. Professor: Eng ${ }^{0}$ Ricardo Cuzziol

Aula 1 - Introdução

Generalidades - áreas de aplicações, cursos, bibliografia

Aula 2 - Tendências no Uso de Polímeros

Aula 3 - Propriedades e Classificação de Polímeros Propriedades Mecânicas

Efeitos de Temperatura / Umidade / Espessura

Anisotropia

Modificação de Propriedades

Aula 4 - Curvas Tensão x Deformação

Fluência / Relaxação

Propriedades Térmicas

Aula 5 - Processos e a Influência nas Propriedades

Mecânicas

Injeção

Sopro

Aula 6 - Design de Peças Plásticas

Espessura

Nervuramento

Cantos Vivos

Montagem

Aula 7 - Bancos de Dados com as Propriedades mecânicas de Polímeros

Aula 8 - Simulação de Processos de Injeção

AUTOMOBILÍSTICA - São privilegiados aspectos relacionados às potencialidades dos programas CAE para solução de problemas de análise estrutural aplicados ao automóvel e componentes. O curso provê considerações sobre análises estáticas, dinâmicas, vibro-acústicas, powertrain e segurança veicular. Também são apresentados aspectos relacionados às oportunidades no mercado de trabalho e consultorias. Os tópicos são acompanhados de exemplos. Duração: 8 h. Professor: Prof. Dr. Renato Vargas

Aula 1 - Generalidades

Áreas de aplicações

Cursos

Bibliografia

Programas Computacionais - restritos / livres

Mercado de trabalho - perspectivas

Aula 2 - Análises Estáticas e Dinâmicas

Análises Vibro-Acústicas

Aula 3 - Otimização

Modificação Material - Plásticos

Aula 4 - Powertrain

Análise térmica acoplada

Análise Multifísica

Aula 5 - Crashworthiness
Aula 6 - Generalidades sobre consultorias

Definição de escopo - identificar desafios

Elaboração da proposta comercial

Execução do cronograma físico-financeiro

ÓLEO E GÁS - São apresentadas potencialidades dos programas CAE para solução de problemas de análise estrutural na área de óleo e gás. $\mathrm{O}$ curso provê considerações sobre análises estáticas, dinâmicas, aplicados ao cálculo de plataformas de petróleo, equipamentos subsea, tubulações, etc.. É introduzido o conceito de simulador de embarcações para estudos de navegabilidade e marinharia. Os tópicos são acompanhados de exemplos. Duração: 4 h. Professor: Eng ${ }^{0}$ Afonso Cabrera

Aula 1 - Generalidades

Exemplos de Aplicações

Cursos

Bibliografia

Mercado de trabalho - perspectivas

Aula 2 - Normas

DNV / ASME / ABS

Design by Rules x Design by Analysis

Aula 3 - Programas Computacionais

Programas Computacionais - restritos / livres

Aula 4 - Generalidades sobre consultorias

Definição de escopo - identificar desafios

Execução do cronograma físico-financeiro

Aula 4 - Descrição de um Caso Real

ESTRUTURAS METÁLICAS - São apresentados aspectos relacionados às potencialidades dos programas de simulação para solução de problemas de análise de estruturas metálicas. O curso provê considerações sobre análises estáticas, dinâmicas, aplicados ao cálculo de estruturas metálicas em geral, pontes, transportadores de esteiras, torres de britagem, equipamentos de mineração e normalização relacionada à área. Os tópicos são acompanhados de exemplos. Duração: 4 h

Aula 1 - Generalidades

Áreas de aplicações - equipamentos de mineração, S11D, Porto de São Luís, Samarco, Anglo American.

Evolução do cálculo estrutural "Cálculo manual" $\mathrm{x}$ Computador

Cursos sobre cálculo de estruturas metálicas Bibliografia

Aula 2 - Cálculo de Estruturas

Disciplinas envolvidas

Importância da Normalização Técnica - AISC / ABNT

Cálculo de uma treliça - Programa Smartsteel Programas Computacionais - restritos / livres

Aula 3 - Descrição de um Caso real Identificar desafios técnicos, ferramentas para solução, normas, aspectos comerciais; 
Como a solução agregou para o cliente: aspectos técnicos, financeiros, metodológicos, manutenção, produtividade

Aula 4 - Generalidades sobre consultorias

Definição de escopo - identificar desafios

Elaboração da proposta comercial

Execução do cronograma físico-financeiro

Mercado de trabalho - perspectivas

TUBULAÇÕES - São apresentados aspectos relacionados às potencialidades dos programas de simulação para cálculo de tubulações e suportação, estudos de flexibilidade, e introduzidas as principais normalizações na área. Os tópicos são acompanhados de exemplos. Duração: 4 h

Aula 1 - Generalidades

Áreas de aplicações com exemplos

Cursos

Bibliografia

Mercado de trabalho - tendências

Aula 2 - Análise Estrutural

Análise de Flexibilidade

Suportes

Aula 3 - Normas

Aula 4 - Programas Computacionais - restritos/livres Exemplo: cálculo de tubulação

Aula 5 - Descrição de um Caso Real - Identificar desafios técnicos, ferramentas para solução, aspectos comerciais. Como a solução agregou para o cliente: aspectos técnicos, financeiros, metodológicos, manutenção, produtividade.

\section{MÓDULO CONTEXTO}

Este módulo foi desenvolvido por meio de entrevistas com consultores e empresários experientes no mercado nacional e professores. Nestas entrevistas são exploradas as trajetórias profissionais e os desafios técnicos em diálogo contínuo com o contexto nacional. O principal objetivo deste Módulo é identificar e discutir as peculiaridades do desenvolvimento de trabalhos de consultorias no Brasil.

- Trajetória Pessoal e Empresarial - influências, contexto sócio-político-econômico

- Principais desafios: comerciais / técnicos / políticos Importância do investimento em conhecimento interação com a Universidade

- Consultoria no Brasil e Contexto Nacional. Diferenças em relação aos outros países

- Oportunidades de mercado na atualidade.

\section{CARACTERÍSTICAS DA PLATAFORMA RELIEF}

A Plataforma possui as seguintes funcionalidades:

- Disponibilização das videoaulas de todos os Módulos
- Acompanhamento da performance do aluno

- Possibilidade de transmissões ao vivo

- Certificação do curso

- Trilha de aprendizagem

- Acesso a artigos disponibilizados pelos professores

- Acesso aos exercícios analíticos desenvolvidos pelos professores

- Acesso aos exercícios tutoriais de simulação numérica com programas livres

- Acesso aos programas desenvolvidos pelos professores

- Acesso aos programas livres
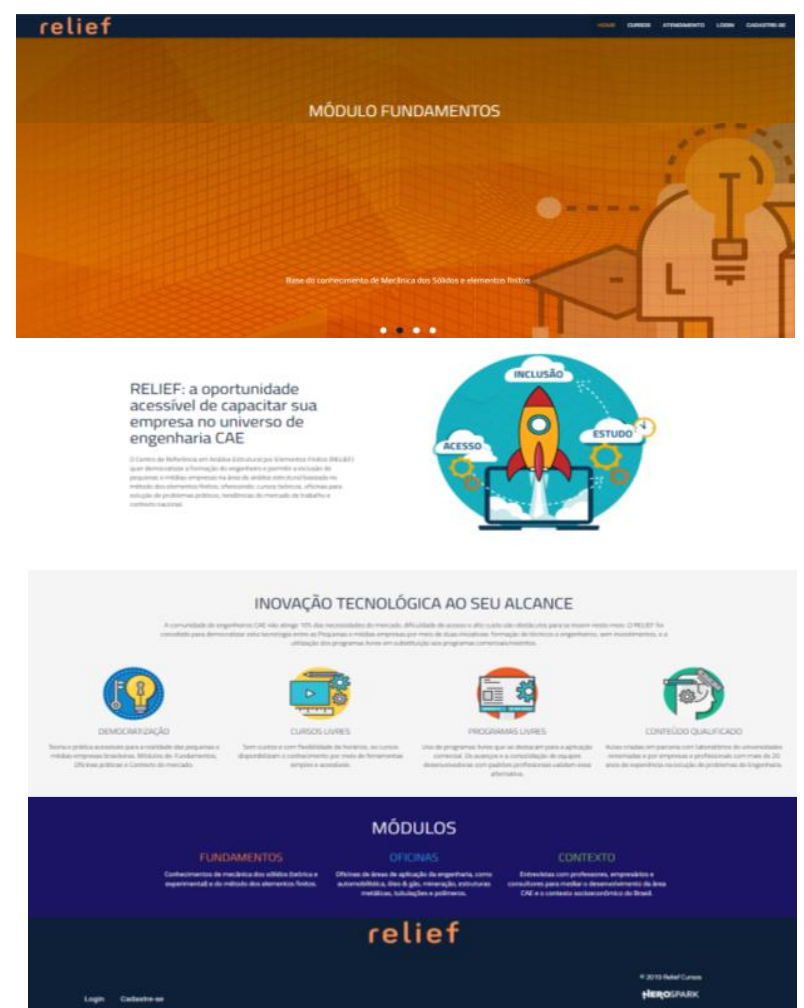

Figura 1 - Home da Plataforma RELIEF

https://reliefcursos.eadbox.com.br/

PROJETO PILOTO - O Projeto Piloto para testes da Plataforma foi desenvolvido no período entre novembro/2019 e abril de 2020 com empresas e profissionais com as características do público alvo. Neste projeto piloto foram testadas todas as funcionalidades da Plataforma e identificadas as eventuais melhorias. Estas empresas e profissionais foram selecionadas junto a rede de contatos do NEP.

\section{APOIOS INSTITUCIONAIS}

EMPRESAS, ASSOCIAÇÕES E ENTIDADES DE CLASSE -Várias empresas de pequeno e médio porte apoiam a Plataforma: Muniz Spada, HBR, Oceânica, Scan Metrologia, Smartsteel, Steel, CHVidom, VIAMOB, GAPE, NANO Tecnologia, etc.. 
O RELIEF concede uma atenção especial às Associações de Classe e Instituições Empresarias, que reúnem um expressivo número de associados que necessitam deste tipo de qualificação. Atualmente, estão em negociação os apoios institucionais com algumas entidades, dentro da perspectiva de cumprir 05 objetivos:

- Disponibilizar a plataforma para os associados.

- As entidades auxiliarem na divulgação da plataforma.

- As instituições identificarem demandas internas para a criação de novos cursos.

- Busca de subsídios para criação de novos cursos.

- A criação de uma rede de apoio institucional para fortalecer a marca do RELIEF e permitir aos seus participantes alunos e colaboradores uma chancela que permita o reconhecimento profissional no mercado.

Várias reuniões foram realizadas com instituições e associações de classe. Abaixo há um descritivo destas instituições.

- Sindicato do Engenheiros de São Paulo (http://www.seesp.org.br/site/) é uma instituição com mais de 60 mil afiliados e provê cursos de aperfeiçoamento e apoio às empresas.

- Abimaq - A Associação Brasileira da Indústria de Máquinas e Equipamentos (http://www.abimaq.org.br/) atua no setor há mais de 80 anos realizando ações junto às instâncias políticas e econômicas. A Abimaq desenvolve ações para aprimorar a capacitação de seus associados. Possui sedes regionais distribuídos pelo País, e representa atualmente cerca de 7.500 empresas dos mais diferentes segmentos fabricantes de bens de capital mecânicos.

- SENAI - (http://www.sp.senai.br/) desenvolve ampla gama de programas de formação profissional.

- Parque Tecnológico de São José dos Campos (http://www.pqtec.org.br/) promove ciência, tecnologia, inovação e estimula a cooperação entre empresas e instituições de pesquisa para a realização de projetos de pesquisa.

- Centro de Integração Empresa - Escola CIEE (https://portal.ciee.org.br/) é uma instituição com mais de 7 milhões de alunos de ensino médio e superior.

UNIVERSIDADES - Alguns laboratórios de Universidades manifestaram o apoio à Plataforma RELIEF. A principal motivação para o apoio é proveniente da qualidade das aulas disponibilizadas na Plataforma e o reconhecimento do valor desta iniciativa para a formação de engenheiros. Por outro lado, várias universidades mantêm cursos de elementos finitos utilizando programas restritos, sob licença educacional. Entretanto, os programas livres são mais apropriados para o aprendizado, porque permitem a implementação de novas formulações e a criação de interfaces, livremente. Com estas premissas foram obtidos os apoios dos seguintes laboratórios:

- Grupo de Análise e Projeto Mecânico (GRANTE) UFSC - O GRANTE (http://www.grante.ufsc.br/)_do Departamento de Engenharia Mecânica da UFSC atua no ensino, pesquisa e extensão. É composto por uma equipe dedicada ao desenvolvimento e utilização de ferramentas numéricas de simulação mecânica na área de Mecânica dos Sólidos: Modelamento Mecânico, Elementos Finitos, Técnicas de Otimização de Projeto, Dano e Fadiga, Mecânica da Fratura, Materiais Compostos, Confiabilidade, dentre outros.

- Laboratório de Metalurgia Física (LAMEF - EMBRAPII) - UFRGS O LAMEF (https://www.ufrgs.br/lamef) é um laboratório da Universidade do Rio Grande do Sul (UFRGS) que possui profunda interação com o setor produtivo, e experiência na execução de serviços tecnológicos e projetos de inovação. O LAMEF é um dos maiores Laboratórios nacionais, com mais de 200 componentes e destaca-se em diversas áreas de atuação, em especial na área Petroquímica.

- Laboratório de Mecânica Estrutural (LaMES) - UTFPR O LaMEs é vinculado ao Centro de Inovação Tecnológica da UTFPR e atua nos seguintes temas: Método dos Elementos Finitos, Otimização Estrutural, Mecânica dos Materiais e Análise Experimental de Tensões. Mantêm projetos de pesquisa financiados por FINEP, CNPq e empresas privadas, WEG, Siemens, Volvo, New Holland, Vale, etc.

- Centro de Competência em Software Livre CCSL - IME /USP.

SUSTENTABILIDADE FINANCEIRA - O custo total da Plataforma RELIEF foi de 265 mil reais (Fapesp = $\mathrm{R} \$ 140.000$ / NEP-Parceiros $=\mathrm{R} \$ 125.000$ )

A sustentabilidade financeira da Plataforma está sendo viabilizada pela empresa NEP Consultoria, mas com movimentos para a consolidação de uma rede de contatos com instituições privadas e entidades de classe. Algumas etapas relativas à sustentação financeira da Plataforma serão realizadas após alguns meses de operação porque requerem uma interação e manifestações do público alvo:

- Solicitação de serviços de suporte para uso dos programas livres especializados.

- Solicitação de serviços de consultorias especializadas.

- Demandas de cursos customizados às necessidades de empresas.

- Desenvolvimento de interfaces para adequar os programas livres às necessidades de problemas de engenharia e desenvolvimento de produtos.

\section{CONCLUSÕES}

A Plataforma RELIEF está disponibilizada conforme a proposta prevista em [1], e atualmente já conta com mais de 100 alunos. A Plataforma mantém-se no mercado como a 
única alternativa viável para as PMEs e engenheiros consultores porque não existem empresas fornecedoras de cursos gratuitos. Assim, o RELIEF é a primeira iniciativa nacional e, provavelmente, internacional, que oferece esta oportunidade para a categoria de engenheiros.

As manifestações sobre a proposta e as avaliações sobre os cursos disponibilizados até o momento são amplamente favoráveis nos diversos setores, desde a Academia, Empresas Privadas, Associações, e Entidades de Classe.Com este impacto inicial é possível perceber que a Plataforma RELEIF já constitui uma realidade de mercado, onde Universidades em Empresas poderão conjugar os interesses e esforços nas soluções de engenharia.

A equipe do RELIEF está formatando a continuidade do Projeto para submissão ao Programa PIPE FASE 2. Nesta próxima fase, os objetivos são a ampliação da Plataforma com mais oficinas, mais disciplinas e divulgação. Estas metas estão vinculadas às manifestações de retorno e críticas dos usuários, para manter a Plataforma conectada ao mercado. Abaixo estão descritas algumas atividades:

- Consolidar Apoios Institucionais - com entidades e associações de classe para estabelecer parcerias e criar novas demandas para ampliação da Plataforma.

- Criação de outras Disciplinas de Fundamentos: Análise Não Linear Impacto Método dos Elementos Finitos não Linear

- Criação de outras Oficinas - Identificar a demanda das Associações, Entidades de Classe e empresas privadas.

- Ampliação do Módulo Contexto - Realização de Novas entrevistas com Profissionais da Área CAE e produção de artigos.

- Divulgação da Plataforma - Será continuada a atividade de visitas regulares às PMEs e participação de eventos para divulgação com apresentações sistemáticas sobre o potencial da Plataforma RELIEF.

\section{BIBLIOGRAFIA}

1. Vargas, R.T. (2017), "CAE com Programas Livres Uma Disruptura em Análise Estrutural”, Artigo SAE 2017-36-0274.

2. Relatório Final do Projeto Pipe Fapesp - 2017-20646-3 (2019), "Criação do Centro de Referência em Análise Estrutural por Elementos Finitos", São Paulo, SP.

3. Salerno, M. S. (2015), "Gestão da Inovação e Competitividade no Brasil: Da Teoria à Prática", Bookman, São Paulo, SP.

4. Ostewalder, A., Pigneur, Y. (2011), "BMG - Business Model Generation - Inovação em Modelos de Negócios,
Alta books Editora, Rio de Janeiro.

5. Alves, M. (2019), "Impact Engineering - Fundamentals, Experiments, Nonlinear Finite Elements", Pre-Print, São Paulo, SP.

6. Bittencourt, M. L. (2014), “Computational Solid Mechanics, CRC Press, Campinas, SP.

\section{INFORMAÇÕES DE CONTATO}

Renato Teixeira Vargas - Engenheiro Mecânico formado na Universidade Federal de Santa Catarina e Doutor pela Universidade de São Paulo. Diretor Técnico da Empresa NEP Consultoria que ministra cursos de executa consultorias na área de análise estrutural por elementos finitos há 15 anos. Desde 2019 é Coordenador da Plataforma RELIEF.

Rua Prof. Oséias Silveira, 35 - CEP 05535-020

São Paulo - SP

renato@nepconsult.com.br

\section{ABREVIAÇÕES}

MEF

Método dos Elementos Finitos

PMEs

Pequenas e Médias Empresas

CAE Computer Aided Engineering

LGPL Lesser General Public License 